\title{
Photolumineszenz und sensibilisierte IR-Emission der dreiwertigen Seltenen Erden in $\mathrm{Ca}_{3} \mathrm{La}_{2} \mathrm{Te}_{2} \mathrm{O}_{12}$
}

\author{
H.-D. Autenrieth, B. Kottmann und S. Kemmler-Sack \\ Institut für Anorganische Chemie der Universität \\ Z. Naturforsch. 40 a, 699-705 (1985); received April 29, 1985 \\ Photoluminescence and Sensitized IR Emission of the Trivalent Rare Earths in $\mathrm{Ca}_{3} \mathrm{La}_{2} \mathrm{Te}_{2} \mathrm{O}_{12}$ \\ By activation of the new host lattice $\mathrm{Ca}_{3} \mathrm{La}_{2} \mathrm{Te}_{2} \mathrm{O}_{12}$ with trivalent rare earth ions an emission in \\ the visible $\left(\mathrm{Ln}^{3+}=\mathrm{Sm}, \mathrm{Eu}, \mathrm{Tb}, \mathrm{Dy}, \mathrm{Ho}, \mathrm{Er}, \mathrm{Tm}\right)$ or near infrared region $(\mathrm{Nd}, \mathrm{Ho}, \mathrm{Er}, \mathrm{Tm}, \mathrm{Yb})$ is \\ observed. Energy transfer from $\mathrm{Nd}^{3+}$ to $\mathrm{Yb}^{3+}$, from $\mathrm{Er}^{3+}, \mathrm{Yb}^{3+}$ to $\mathrm{Ho}^{3+}$ and from $\mathrm{Yb}^{3+}$ to $\mathrm{Tm}^{3+}$ \\ has been found to occur. The excitation, emission and diffuse reflectance spectra are analyzed.
}

\section{Einführung}

Im Rahmen von Untersuchungen über die Lumineszenz $\mathrm{Te}^{6+}$-haltiger Oxide $[1-3]$ wurde bei Aktivierung des neuen, im $\mathrm{Ca}_{5} \mathrm{Re}_{2} \mathrm{O}_{12}$-Typ [4] kristallisierenden Wirtsgitters $\mathrm{Ca}_{3} \mathrm{La}_{2} \mathrm{Te}_{2} \mathrm{O}_{12}$ [1] mit einigen dreiwertigen Seltenen Erden (Ln) eine Emission im sichtbaren Gebiet und/oder nahen IR beobachtet. Durch Einbau weiterer, als Sensibilisator wirkender Selten Erd-Ionen ( $\mathrm{Ln}^{\prime}$ ) läßt sich die IREmission verstärken. Im folgenden werden die Anregungs-, Emissions- und diffusen Reflexionsspektren von $\mathrm{Ca}_{3} \mathrm{La}_{2} \mathrm{Te}_{2} \mathrm{O}_{12}: \mathrm{Ln}$ sowie $\mathrm{Ca}_{3} \mathrm{La}_{2} \mathrm{Te}_{2} \mathrm{O}_{12}: \mathrm{Ln}$, Ln' diskutiert.

\section{Experimentelle Angaben}

Zur Darstellung von $\mathrm{Ca}_{3} \mathrm{La}_{2} \mathrm{Te}_{2} \mathrm{O}_{12}$ sowie der dotierten Verbindungen $\mathrm{Ca}_{3} \mathrm{La}_{2-(x+y)} \mathrm{Ln}_{x} \mathrm{Ln}_{y}^{\prime} \mathrm{Te}_{2} \mathrm{O}_{12}$ (Aktivator: Ln; Sensibilisator: Ln'; im folgenden mit CaT:Ln $(y=0)$ bzw. CaT:Ln, Ln' abgekürzt; bei Verwendung von zwei bzw. drei Sensibilisatoren werden diese nacheinander angeführt und ihre Konzentration mit $y, y^{\prime}$ bzw. $y^{\prime \prime}$ bezeichnet) wurde wie unter [1] verfahren. Ausgangsprodukte für die Seltenen Erden waren die Oxide $\mathrm{Ln}_{2} \mathrm{O}_{3}(\mathrm{Ln}=\mathrm{Nd}, \mathrm{Sm}$, $\mathrm{Eu}, \mathrm{Dy}, \mathrm{Ho}, \mathrm{Er}, \mathrm{Tm}, \mathrm{Yb})$ und $\mathrm{LnO}_{1,75}(\mathrm{Ln}=\mathrm{Pr}, \mathrm{Tb}$; jeweils 99,9\%; Rasmus u. Co). Die Verbindungen CaT:Ln und CaT:Ln,Ln' sind weiß oder zeigen ganz schwach die Farben der entsprechenden Ln-

Reprint requests to Herrn Prof. Dr. S. Kemmler-Sack, Institut für Anorganische Chemie der Universität Tübingen, Auf der Morgenstelle 18, D-7400 Tübingen. bzw. Ln'-Ionen. Die Gitterkonstanten (Pulverdiffraktometer von Philips, CuK $x$-Strahlung) liegen jeweils nahe bei den Werten des reinen Wirtsgitters $(a=9,79(1) ; c=55,5(1) \AA[1]) ;$ Analysendaten und Pyknometerdichten (Sperrflüssigkeit n-Oktan; $22,2^{\circ} \mathrm{C}$ ) stimmen mit den berechneten Werten überein (z. B. CaT: Te[\%]=30,1 (gef.); 30,19 (ber.); $\varrho\left(\mathrm{gcm}^{-3}\right)=5,25$ (gef.); 5,482 (ber.)). Die spektroskopischen Untersuchungen wurden wie unter [5] durchgeführt; an Stelle des RCA-Multipliers 1P28 fand der Typ 4832 Verwendung.

\section{Ergebnisse}

\subsection{Lumineszenz im sichtbaren Gebiet}

Die aktivierten Verbindungen CaT:Ln zeigen ebenso wie das reine Wirtsgitter eine starke optische Absorption, welche bei $\sim 35000 \mathrm{~cm}^{-1}$ einsetzt und einem charge transfer (CT) vom Sauerstoff zum Tellur innerhalb der oktaedrischen $\mathrm{TeO}_{6}$-Gruppen des Wirtsgitter entspricht. Bei Anregung in die Bandkante $\left(\sim 38000 \mathrm{~cm}^{-1}\right)$ wird für CaT: Ln weder bei Raumtemperatur (RT) noch bei $77 \mathrm{~K}$ eine Emission der $\mathrm{TeO}_{6}$-Gruppen registriert.

Bei Ln-Aktivierung treten für $\mathrm{Ln}^{3+}=\mathrm{Sm}, \mathrm{Eu}, \mathrm{Tb}$, Dy, Ho, Er Emissionen im sichtbaren Gebiet auf, welche $4 \mathrm{f} \rightarrow 4 \mathrm{f}$-Übergängen in den jeweiligen $\mathrm{Ln}^{3+}$. Termsystemen zuzuordnen sind. Die Lumineszenzfarben finden sich gemeinsam mit den intensivsten Emissionsmaxima in Tabelle 1. Die mit $\operatorname{Pr}^{3+}$ dotierte Verbindung zeigt keine nennenswerte Emission.

In den Anregungsspektren von CaT: Ln treten mit Ausnahme von $\mathrm{Ln}^{3+}=\mathrm{Eu}$ sowohl bei RT als auch $77 \mathrm{~K}$ allein $4 \mathrm{f} \rightarrow 4 \mathrm{f}$-Übergänge auf; das jeweils

0340-4811 / 85 / 0700-0699 \$ 01.30/0. - Please order a reprint rather than making your own copy. 
intensivste Anregungsmaximum zeigt Tabelle 1. Für CaT: Eu läßt sich neben den Übergängen innerhalb der Konfiguration $4 \mathrm{f}^{6}$ eine breite Bande bei $\sim 33000 \mathrm{~cm}^{-1}$ erkennen, die dem CT vom Sauerstoff zum $\mathrm{Eu}^{3+}$ entspricht. Bei $77 \mathrm{~K}$ hat gegenüber RT die Intensität aller Anregungsbanden, die von dem dem Grundzustand $\left({ }^{7} \mathrm{~F}_{0}\right)$ eng benachbarten Term ${ }^{7} F_{1}$ ausgehen, abgenommen, da dessen thermische Besetzung zurückgegangen ist.

Das Emissionsspektrum von CaT: Eu zeigt neben den intensiveren Übergängen ${ }^{5} \mathrm{D}_{0} \rightarrow{ }^{7} \mathrm{~F}_{\mathrm{J}}$ schwächere Banden vom Typ ${ }^{5} \mathrm{D}_{2},{ }^{5} \mathrm{D}_{1} \rightarrow{ }^{7} \mathrm{~F}_{\mathrm{J}}$, deren relative Intensität $\left(I_{\text {rel }}\right)$ mit steigender Eu-Konzentration aufgrund einer Kreuzrelaxation sinkt. Für $\mathrm{x}=$ const. wird bei Anregung in den CT gegenüber $4 \mathrm{f}$-Einstrahlung ebenfalls eine Intensitätsverminderung der Gruppe ${ }^{5} \mathrm{D}_{2},{ }^{5} \mathrm{D}_{1} \rightarrow{ }^{7} \mathrm{~F}_{\mathrm{J}}$ beobachtet, was bei einem energetisch relativ niedrig liegenden $\mathrm{CT}$ zu erwarten ist [6]. Aus den beobachteten Auswahlregeln $\left({ }^{5} \mathrm{D}_{0} \rightarrow{ }^{7} \mathrm{~F}_{0}\right.$ als Singulett beobachtbar, ${ }^{5} \mathrm{D}_{0} \rightarrow{ }^{7} \mathrm{~F}_{1}$ zum Triplett aufgespalten, ${ }^{5} \mathrm{D}_{0} \rightarrow{ }^{7} \mathrm{~F}_{2}$ dominierend) geht für $\mathrm{Eu}^{3+}$ die Besetzung einer Lage der Symmetrie $C_{s}, C_{n}$ oder $C_{n v}$ hervor. In Übereinstimmung steht die Strukturuntersuchung, welche für $\mathrm{Eu}^{3+}$ eine Lage der Symmetrie $C_{3 v}$ ergibt [4].

Bei $77 \mathrm{~K}$ steigt die relative Emissionsintensität $\left(I_{\text {rel }}\right)$ für $\mathrm{Ln}^{3+}=\mathrm{Sm}, \mathrm{Eu}, \mathrm{Tb}, \mathrm{Dy}, \mathrm{Tm}$ an. Mit $\mathrm{Ln}^{3+}=$ Ho, Er trifft dies im Gebiet von $18500 \mathrm{~cm}^{-1}$ allein auf die Übergänge ${ }^{5} \mathrm{~S}_{2} \rightarrow{ }^{5} \mathrm{I}_{8}$ bzw. ${ }^{4} \mathrm{~S}_{3 / 2} \rightarrow{ }^{4} \mathrm{I}_{15 / 2}$ $\mathrm{zu}$, während die thermische Besetzung des energetisch knapp oberhalb von ${ }^{5} \mathrm{~S}_{2}$ bzw. ${ }^{4} \mathrm{~S}_{3 / 2}$ angesiedelten Terme ${ }^{5} \mathrm{~F}_{4}$ (Ho) bzw. ${ }^{2} \mathrm{H}_{11 / 2}$ (Er) zurückgegangen ist, wodurch $I_{\text {rel }}$ von ${ }^{5} \mathrm{~F}_{4} \rightarrow{ }^{5} \mathrm{I}_{8}$ bzw. ${ }^{2} \mathrm{H}_{11 / 2} \rightarrow{ }^{4} \mathrm{I}_{15 / 2}$ sinkt.

\subsection{Lumineszenz im nahen IR}

Bei Aktivierung von $\mathrm{CaT}$ mit $\mathrm{Ln}^{3+}=\mathrm{Nd}, \mathrm{Ho}, \mathrm{Er}$, $\mathrm{Tm}, \mathrm{Yb}$ treten Emissionen im nahen IR auf. Die Bandenlagen und ihre Zuordnung zeigt Tabelle 2 gemeinsam mit den jeweils intensivsten Anregungsmaxima. Die stärksten Emissionsmaxima lassen sich bei breitbandiger Anregung in den diffusen Reflexionsspektren beobachten $\left(\mathrm{Nd}^{3+}:{ }^{4} \mathrm{~F}_{3 / 2} \rightarrow{ }^{4} \mathrm{I}_{13 / 2}\right.$, ${ }^{4} \mathrm{I}_{11 / 2}, \quad{ }^{4} \mathrm{I}_{9 / 2} ; \quad \mathrm{Ho}^{3+}: \quad{ }^{5} \mathrm{I}_{7} \rightarrow{ }^{5} \mathrm{I}_{8} ; \quad \mathrm{Er}^{3+}:{ }^{4} \mathrm{I}_{13 / 2} \rightarrow{ }^{4} \mathrm{I}_{15 / 2} ;$ $\mathrm{Tm}^{3+}:{ }^{3} \mathrm{H}_{4} \rightarrow{ }^{3} \mathrm{H}_{6}$; (Termbezeichnung hier und im folgenden nach [7, 8]); $\mathrm{Yb}^{3+}:{ }^{2} \mathrm{~F}_{5 / 2} \rightarrow{ }^{2} \mathrm{~F}_{7 / 2}$; Abb. 1).

Für die sowohl im sichtbaren Gebiet als auch im nahen IR emittierenden Aktivatoren $\mathrm{Ho}^{3+}$ und $\mathrm{Er}^{3+}$ stimmt das intensivste Anregungsmaximum für die Emissionen in beiden Spektralbereichen überein
Tab. 1. Lumineszenzfarben und intensivste $4 \mathrm{f} \rightarrow 4 \mathrm{f}$-Übergänge im System CaT: Ln (RT).

\begin{tabular}{lllll}
\hline $\mathrm{Ln}^{3+}$ & $\begin{array}{l}\text { Lumines- } \\
\text { zenzfarbe }\end{array}$ & \multicolumn{2}{l}{$4 \mathrm{f} \rightarrow 4 \mathrm{f}$-Übergang } \\
\cline { 3 - 5 } & & Emission & Anregung \\
\hline $\mathrm{Sm}$ & orange & ${ }^{4} \mathrm{G}_{5 / 2} \rightarrow{ }^{6} \mathrm{H}_{7 / 2}$ & ${ }^{6} \mathrm{H}_{5 / 2} \rightarrow{ }^{4} \mathrm{~L}_{13 / 2}$ \\
$\mathrm{Eu}$ & rot & ${ }^{5} \mathrm{D}_{0}$ & $\rightarrow{ }^{7} \mathrm{~F}_{2}$ & ${ }^{7} \mathrm{~F}_{0} \rightarrow{ }^{5} \mathrm{~L}_{6}$ \\
$\mathrm{~Tb}$ & grün & ${ }^{5} \mathrm{D}_{4}$ & $\rightarrow{ }^{7} \mathrm{~F}_{5}$ & ${ }^{7} \mathrm{~F}_{6} \rightarrow{ }^{5} \mathrm{G}_{6}$ \\
$\mathrm{Dy}$ & gelb & ${ }^{4} \mathrm{~F}_{9 / 2} \rightarrow{ }^{6} \mathrm{H}_{13 / 2}$ & ${ }^{6} \mathrm{H}_{15 / 2} \rightarrow{ }^{4} \mathrm{~F}_{7 / 2}$ \\
$\mathrm{Ho}$ & grün & ${ }^{5} \mathrm{~S}_{2},{ }^{5} \mathrm{~F}_{4} \rightarrow{ }^{5} \mathrm{I}_{8}$ & ${ }^{5} \mathrm{I}_{8} \rightarrow{ }^{5} \mathrm{G}_{6},{ }^{3} \mathrm{~K}_{8}$ \\
Er & grün & ${ }^{4} \mathrm{~S}_{3 / 2} \rightarrow{ }^{4} \mathrm{I}_{15 / 2}$ & ${ }^{4} \mathrm{I}_{15 / 2} \rightarrow{ }^{4} \mathrm{G}_{11 / 2}$ \\
$\mathrm{Tm}$ & blau & ${ }^{1} \mathrm{G}_{4} \rightarrow \rightarrow{ }^{3} \mathrm{H}_{6}$ & ${ }^{3} \mathrm{H}_{6} \rightarrow{ }^{1} \mathrm{D}_{2}$ \\
\hline
\end{tabular}

Tab. 2. IR-Emission bei RT $\left(\mathrm{cm}^{-1}\right)$ und ihre intensivsten Anregungsmaxima.

\begin{tabular}{|c|c|c|c|}
\hline \multirow[t]{2}{*}{$\mathrm{Ln}^{3+}$} & \multicolumn{2}{|c|}{ Emissionen } & \multirow{2}{*}{$\begin{array}{l}\text { intensivste } \\
\text { Anregungsmaxima }\end{array}$} \\
\hline & $\begin{array}{l}\text { Banden- } \\
\text { lage }\end{array}$ & Zuordnung & \\
\hline $\mathrm{Nd}$ & $\begin{array}{c}7300 \\
9300 \\
10900^{\mathrm{a}}\end{array}$ & $\left.\begin{array}{l}{ }^{4} \mathrm{~F}_{3 / 2} \rightarrow{ }^{4} \mathrm{I}_{13 / 2} \\
{ }^{4} \mathrm{~F}_{3 / 2} \rightarrow{ }^{4} \mathrm{I}_{11 / 2} \\
{ }^{4} \mathrm{~F}_{3 / 2} \rightarrow{ }^{4} \mathrm{I}_{9 / 2}\end{array}\right]$ & $\left\{\begin{array}{l}{ }^{4} \mathrm{I}_{9 / 2} \rightarrow{ }^{4} \mathrm{~F}_{5 / 2},{ }^{2} \mathrm{H}_{9 / 2} \\
{ }^{4} \mathrm{I}_{9 / 2} \rightarrow{ }^{4} \mathrm{~F}_{7 / 2},{ }^{4} \mathrm{~S}_{3 / 2}\end{array}\right.$ \\
\hline Ho & $\begin{array}{r}5000 \\
8500 \\
10900 \\
13200\end{array}$ & $\left.\begin{array}{ll}{ }^{5} \mathrm{I}_{7} & \rightarrow{ }^{5} \mathrm{I}_{8} \\
{ }^{5} \mathrm{I}_{6} & \rightarrow{ }^{5} \mathrm{I}_{8} \\
{ }^{5} \mathrm{I}_{5} & \rightarrow{ }^{5} \mathrm{I}_{8} \\
{ }^{5} \mathrm{I}_{4} & \rightarrow{ }^{5} \mathrm{I}_{8}\end{array}\right]$ & ${ }^{5} \mathrm{I}_{8} \rightarrow{ }^{5} \mathrm{G}_{6},{ }^{3} \mathrm{~K}_{8},{ }^{5} \mathrm{~F}_{2}$ \\
\hline Er & $\begin{array}{r}6500 \\
10000 \\
15200\end{array}$ & $\left.\begin{array}{l}{ }^{4} \mathrm{I}_{13 / 2} \rightarrow{ }^{4} \mathrm{I}_{15 / 2} \\
{ }^{4} \mathrm{I}_{11 / 2} \rightarrow{ }^{4} \mathrm{I}_{15 / 2} \\
{ }^{4} \mathrm{~F}_{9 / 2} \rightarrow{ }^{4} \mathrm{I}_{15 / 2}\end{array}\right]$ & ${ }^{4} \mathrm{I}_{15 / 2} \rightarrow{ }^{4} \mathrm{G}_{11 / 2}$ \\
\hline $\operatorname{Tm}^{\mathrm{b}}$ & $\begin{array}{l}5500 \\
6800\end{array}$ & $\left.\begin{array}{r}{ }^{3} \mathrm{H}_{4} \rightarrow{ }^{3} \mathrm{H}_{6} \\
{ }^{3} \mathrm{~F}_{4} \rightarrow{ }^{3} \mathrm{H}_{4}\end{array}\right]$ & 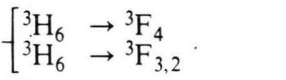 \\
\hline $\mathrm{Yb}$ & $9900^{\mathrm{a}}$ & ${ }^{2} \mathrm{~F}_{5 / 2} \rightarrow{ }^{2} \mathrm{~F}_{7 / 2}$ & ${ }^{2} \mathrm{~F}_{7 / 2} \rightarrow{ }^{2} \mathrm{~F}_{5 / 2}$ \\
\hline
\end{tabular}

a Aus den diffusen Reflexionsspektren.

b Termbezeichnung nach $[7,8]$.

(vgl. Tab. 1 und 2 sowie Abbildung 2). Dagegen ist bei $\mathrm{Ln}^{3+}=\mathrm{Tm}$ die blaue Emission ${ }^{1} \mathrm{G}_{4} \rightarrow{ }^{3} \mathrm{H}_{6}$ über ${ }^{3} \mathrm{H}_{6} \rightarrow{ }^{1} \mathrm{D}_{2}$ anregbar, während $I_{\text {rel }}$ für die IR-Emission bei Einstrahlung in die eng benachbarten Übergänge ${ }^{3} \mathrm{H}_{6} \rightarrow{ }^{3} \mathrm{~F}_{4,3,2}$ gegenüber ${ }^{3} \mathrm{H}_{6} \rightarrow{ }^{1} \mathrm{D}_{2}$ um etwa den Faktor 15 steigt (s. das Anregungsspektrum von $\mathrm{CaT}$ : Tm in Abbildung 2). Bei Temperaturerniedrigung $(77 \mathrm{~K})$ geht die Intensität der IR-Emissionen in allen Fällen zurück.

\subsection{Sensibilisierte Emission im nahen IR}

Werden in das Wirtsgitter CaT neben den Aktivatoren $(\mathrm{Ln})$ eine zweite Sorte an dreiwertigen Seltenen Erden ( $\left.\mathrm{Ln}^{\prime}\right)$ eingebaut, läßt sich für die 


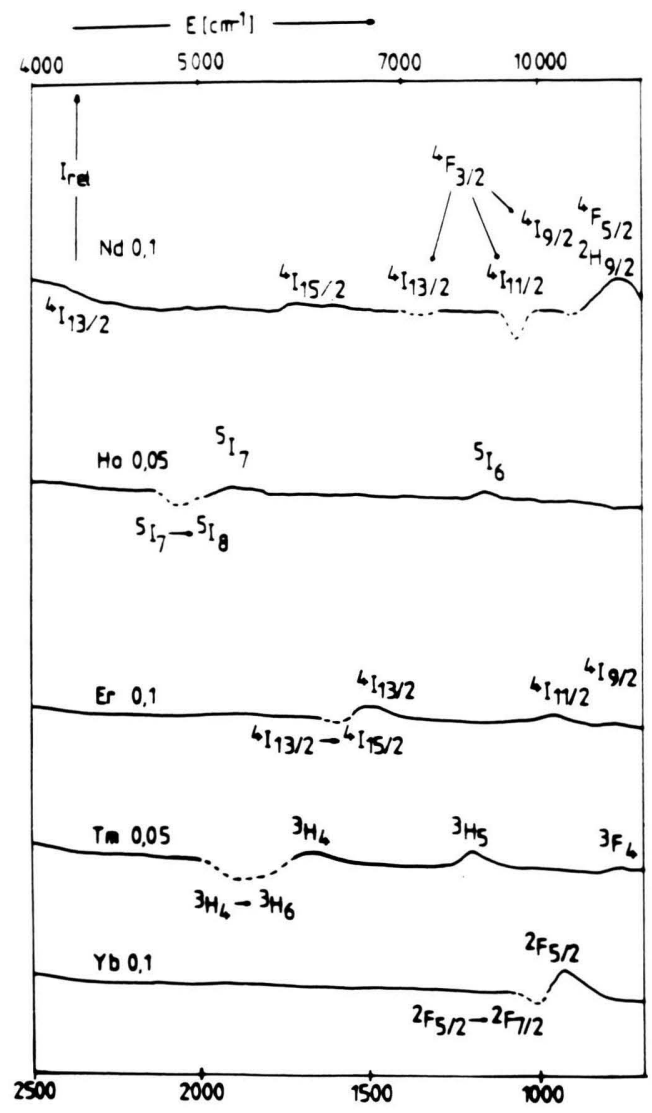

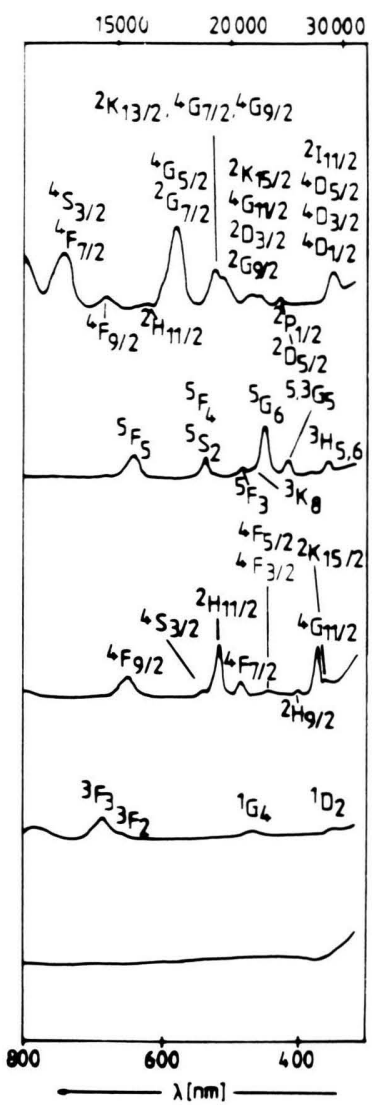

Abb. 1. Diffuse Reflexionsspektren von CaT: Nd $(x=0,1$; Grundzustand ${ }^{4} \mathrm{I}_{9 / 2} ; \mathrm{CaT}: \mathrm{Ho}\left(x=0,05 ;{ }^{5} \mathrm{I}_{8}\right) ; \mathrm{CaT}: \mathrm{Er}$ $\left(x=0,1 ;{ }^{4} \mathrm{I}_{152}\right) ; \mathrm{CaT}: \operatorname{Tm}(x=0,05$; $\left.{ }^{3} \mathrm{H}_{6}\right)$ und $\mathrm{CaT}: \mathrm{Yb}\left(x=0,1 ;{ }^{2} \mathrm{~F}_{7 / 2}\right), \mathrm{Re}$ lative Intensität $\left(I_{\text {rel }}\right)$ in Abhängigkeit von $\lambda(\mathrm{nm})$; -.-.: Emissionen.
Ln/Ln'-Kombinationen Ho/Er; Ho/Tm; Ho/Yb; $\mathrm{Tm} / \mathrm{Yb} ; \mathrm{Yb} / \mathrm{Nd}$ sowie $\mathrm{Ho} / \mathrm{Er}, \mathrm{Yb}$ und $\mathrm{Ho} / \mathrm{Er}, \mathrm{Tm}, \mathrm{Yb}$ eine Sensibilisierung der IR-Emission von Ln herbeiführen.

Die für CaT: $\mathrm{Ln}, \mathrm{Ln}^{\prime}$ bei breitbandiger Einstrahlung gegenüber $\mathrm{CaT}: \mathrm{Ln}$ zu erzielende Intensitätssteigerung (Abb. 3; Faktoren sind jeweils vermerkt) geht aus einem Vergleich mit den diffusen Reflexionsspektren der Abb. 1 (CaT:Ln) hervor, welche mit derselben Verstärkung registriert wurden. Gegenüber CaT:Ho steigt bei gleicher Aktivatorkonzentration $(x=0,05) I_{\text {rel }}$ für die Kombination $\mathrm{Ho} / \mathrm{Er}$ und $\mathrm{Ho} / \mathrm{Yb}$ um etwa den Faktor 4; sowie für $\mathrm{Ho} / \mathrm{Tm}$ um etwa 3 (bei letzterem blieb der zusätzlich vorhandene Anteil an $\mathrm{Tm}^{3+}$-Emission ${ }^{3} \mathrm{H}_{4} \rightarrow{ }^{3} \mathrm{H}_{6}$ unberücksichtigt; vgl. das Emissionsspektrum in Abbildung 5). Die Sensibilisierung der Tm-Emission $(x=0,05)$ durch $\mathrm{Yb}^{3+}$ führt zu einer Verstärkung um etwa den Faktor 2 und bei der Kombination $\mathrm{Yb} / \mathrm{Nd}$ gegenüber $\mathrm{CaT}: \mathrm{Yb}(x$ jeweils 0,1$)$ um den Faktor 6. Eine weitere Steigerung der Sensibilisierungsrate bei breitbandiger Anregung um etwa den Faktor 5,5 bzw. 6,5 ist durch gleichzeitige Kombination des Aktivators $\mathrm{Ho}^{3+}$ mit zwei $(\mathrm{Er}, \mathrm{Yb})$ bzw. drei Sensibilisatoren (Er, Tm, Yb) zu erzielen.

Auskünfte über die bei der Sensibilisierung stattfindenden Energieübertragungsprozesse liefern die Anregungs- und Emissionsspektren.

In den Anregungsspektren der intensivsten Aktivatoremission treten für alle $\mathrm{Ln} / \mathrm{Ln}^{\prime}$-Kombinationen zu den Anregungsmaxima des Aktivators die Sensibilisatorbanden hinzu. In Abb. 4 sind als Beispiel die Anregungsspektren von $\mathrm{CaT}: \mathrm{Ho}, \mathrm{Yb}$ und $\mathrm{CaT}: \mathrm{Ho}, \mathrm{Er}, \mathrm{Tm}, \mathrm{Yb}$ angegeben (Anregungsspektren von CaT:Ho, CaT:Er, CaT:Tm in Abbildung 2). Für CaT:Ho, Yb, das eine vierfach größere Sensibilisatorkonzentration besitzt $(x=0,05 ; y=0,2)$, zeigt der $\mathrm{Yb}^{3+}$-Übergang ${ }^{2} \mathrm{~F}_{5 / 2} \rightarrow{ }^{2} \mathrm{~F}_{7 / 2}$ bei $\sim 10900 \mathrm{~cm}^{-1}$ eine höhere Intensität als die Ho-Banden. Bei $\mathrm{CaT}: \mathrm{Ho}, \mathrm{Er}, \mathrm{Tm}, \mathrm{Yb}$, das sowohl den Aktivator als 


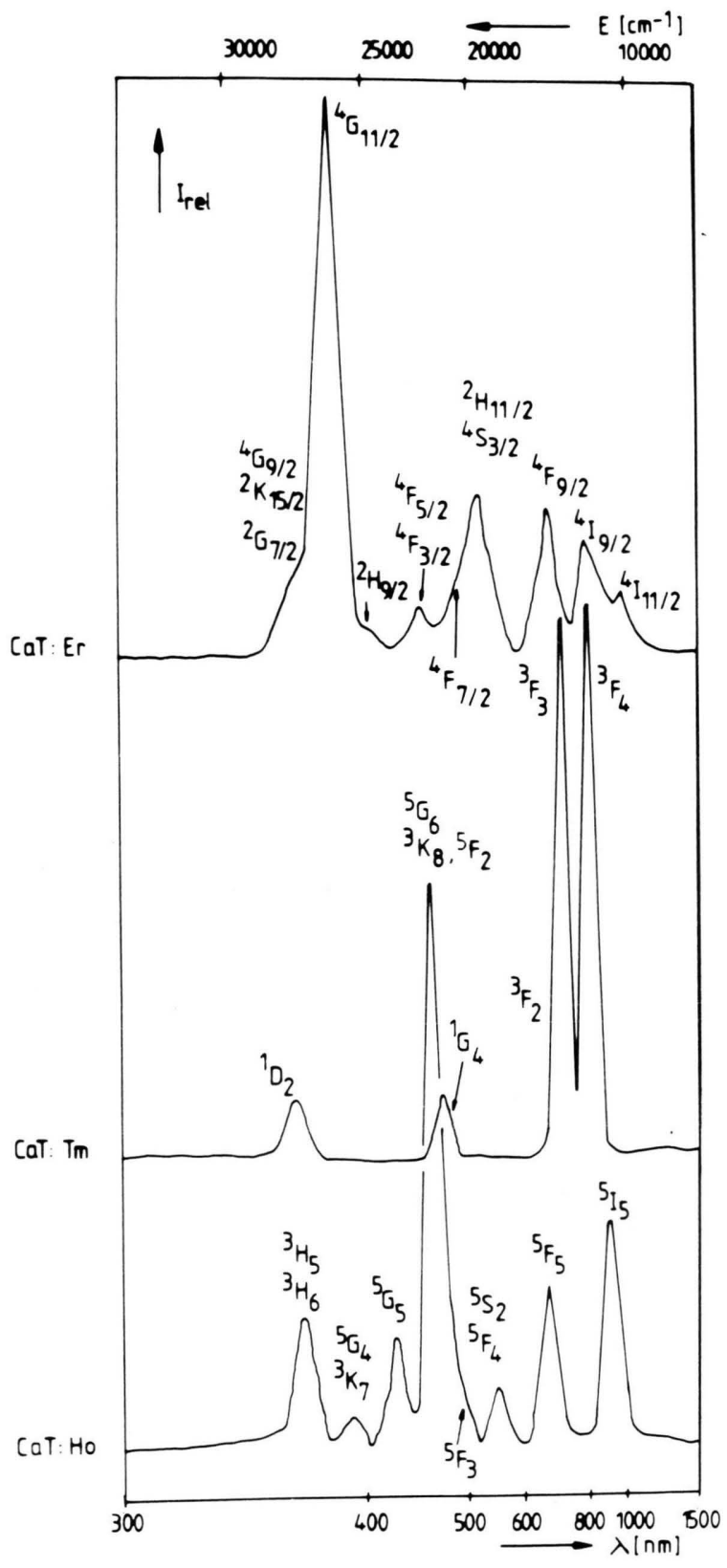

Abb. 2. Anregungsspektren von $\mathrm{CaT}: \mathrm{Er} \quad\left(x=0,1 ; v_{\mathrm{E}} \sim\right.$ $6500 \mathrm{~cm}^{-1}$; Grundzustand $\left.{ }^{4} \mathrm{I}_{15 / 2}\right)$; CaT: Ho $\left(x=0,05 ; v_{\mathrm{E}} \sim\right.$ $\left.5000 \mathrm{~cm}^{-1} ;{ }^{5} \mathrm{I}_{8}\right)$ und CaT:Tm $\left(x=0,05 ; v_{\mathrm{E}} \sim 5500 \mathrm{~cm}^{-1}\right.$; $\left.{ }^{3} \mathrm{H}_{6}\right)$.

auch die Sensibilisatoren in gleicher Konzentration enthält $\left(x=0,05 ; y=y^{\prime}=y^{\prime \prime}=0,05\right)$, besteht das Anregungsspektrum aus einer dichten Bandenfolge, welche einen Bereich von $\sim 28000-10000 \mathrm{~cm}^{-1}$ überstreicht.

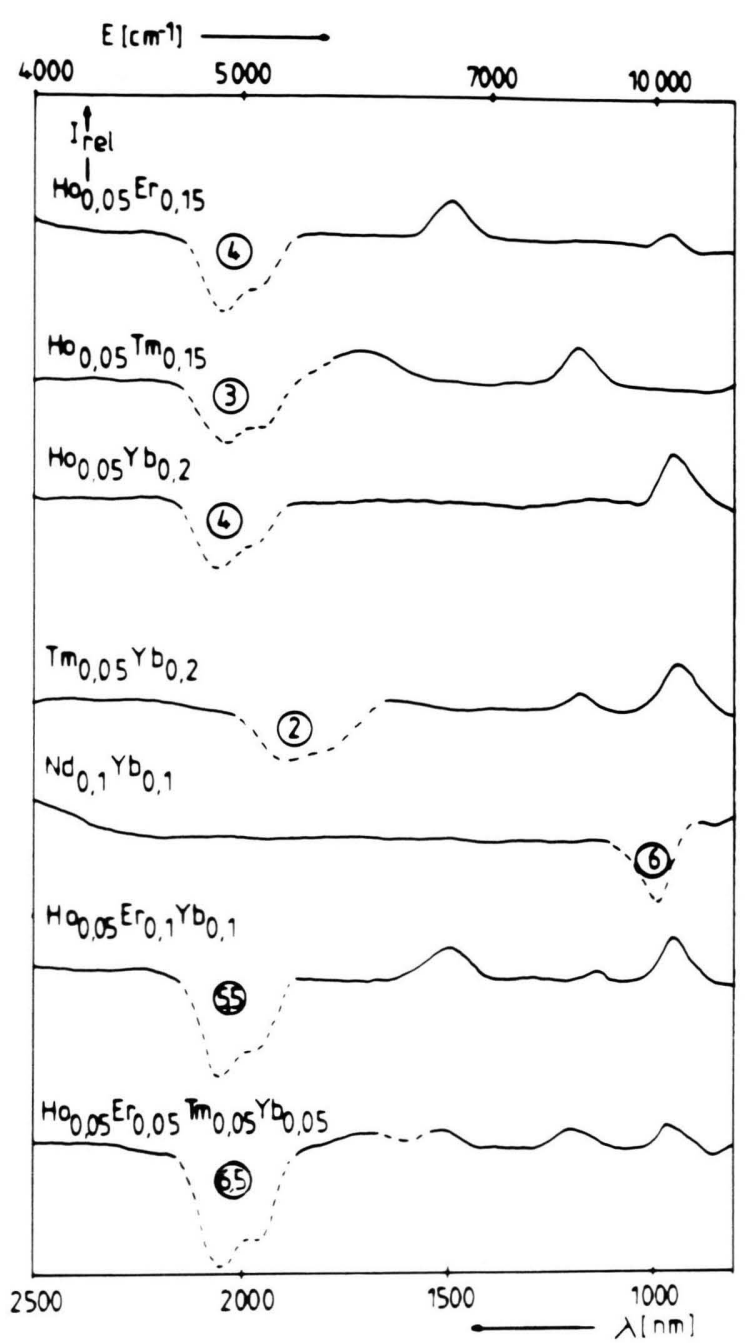

Abb. 3. Ausschnitt aus den diffusen Reflexionsspektren von CaT: Ho, $\operatorname{Er}(x=0,05 ; y=0,15) ; \mathrm{CaT}: \mathrm{Ho}, \mathrm{Tm}(x=$ $0,05 ; y=0,15)$; CaT:Ho, Yb $(x=0,05 ; y=0,2)$; CaT:Tm, $\mathrm{Yb}(x=0,05 ; y=0,2)$; CaT: Yb, Nd $(x=y=0,1)$; CaT:Ho, $\mathrm{Er}, \mathrm{Yb}\left(x=0,05 ; y=y^{\prime}=0,1\right)$ und CaT:Ho, Er, Tm, Yb $\left(x=0,05 ; \quad y=y^{\prime}=y^{\prime \prime}=0,05\right) ;---$ : Absorptionen; -..-: Emissionen (Zunahme von $I_{\text {rel }}$ gegenüber CaT: $L n$ für gleiches $x$ ist jeweils vermerkt). Gleicher Maßstab wie in Abbildung 1 .

Bei Einstrahlung in das intensivste SensibilisatorAnregungsmaximum wird für die Kombinationen $\mathrm{Yb} / \mathrm{Nd}, \mathrm{Ho} / \mathrm{Yb}$ und $\mathrm{Tm} / \mathrm{Yb}$ ausschließlich eine Aktivator-Emission beobachtet, während für $\mathrm{Ho} / \mathrm{Er}$ und $\mathrm{Ho} / \mathrm{Tm}$ neben einer intensiven Aktivator-Emission mit geringerer Intensität auch Banden des Sensibilisators $\mathrm{Er}^{3+}$ bzw. $\mathrm{Tm}^{3+}$ auftreten. In Abb. 5 sind 


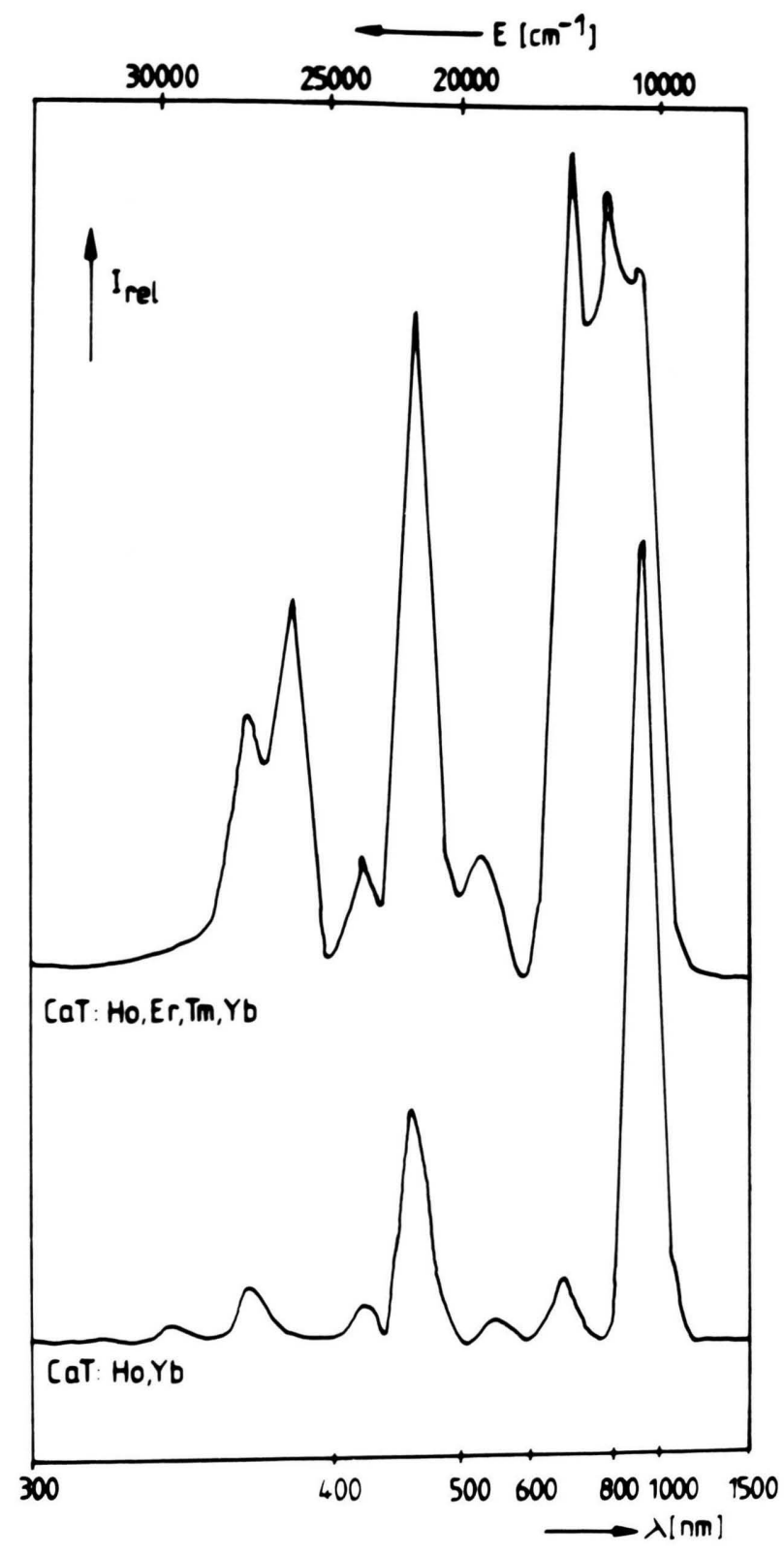

Abb. 4. Anregungsspektren von $\mathrm{CaT}: \mathrm{Ho}, \mathrm{Yb} \quad(x=0,05$; $\left.y=0,2 ; v_{\mathrm{E}} \sim 5000 \mathrm{~cm}^{-1}\right)$ und CaT: $\mathrm{Ho}, \mathrm{Er}, \mathrm{Tm}, \mathrm{Yb}(x=0,05$; $y=y^{\prime}=y^{\prime \prime}=0,05 ; v_{\mathrm{E}} \sim 5000 \mathrm{~cm}^{-1}$ ).

die Emissionsspektren von $\mathrm{CaT}: \mathrm{Ho}, \mathrm{CaT}: \mathrm{Tm}$ sowie $\mathrm{CaT}: \mathrm{Ho}, \mathrm{Tm}$ und $\mathrm{CaT}: \mathrm{Tm}, \mathrm{Yb}$ gegenübergestellt. Für eine Kombination der Sensibilisatoren $\mathrm{Er}^{3+}$ und $\mathrm{Yb}^{3+}(\mathrm{CaT}: \mathrm{Ho}, \mathrm{Er}, \mathrm{Yb})$ bzw. $\mathrm{Er}^{3+}, \mathrm{Tm}^{3+}$ und $\mathrm{Yb}^{3+}(\mathrm{CaT}: \mathrm{Ho}, \mathrm{Er}, \mathrm{Tm}, \mathrm{Yb})$ ergibt sich bei selek-

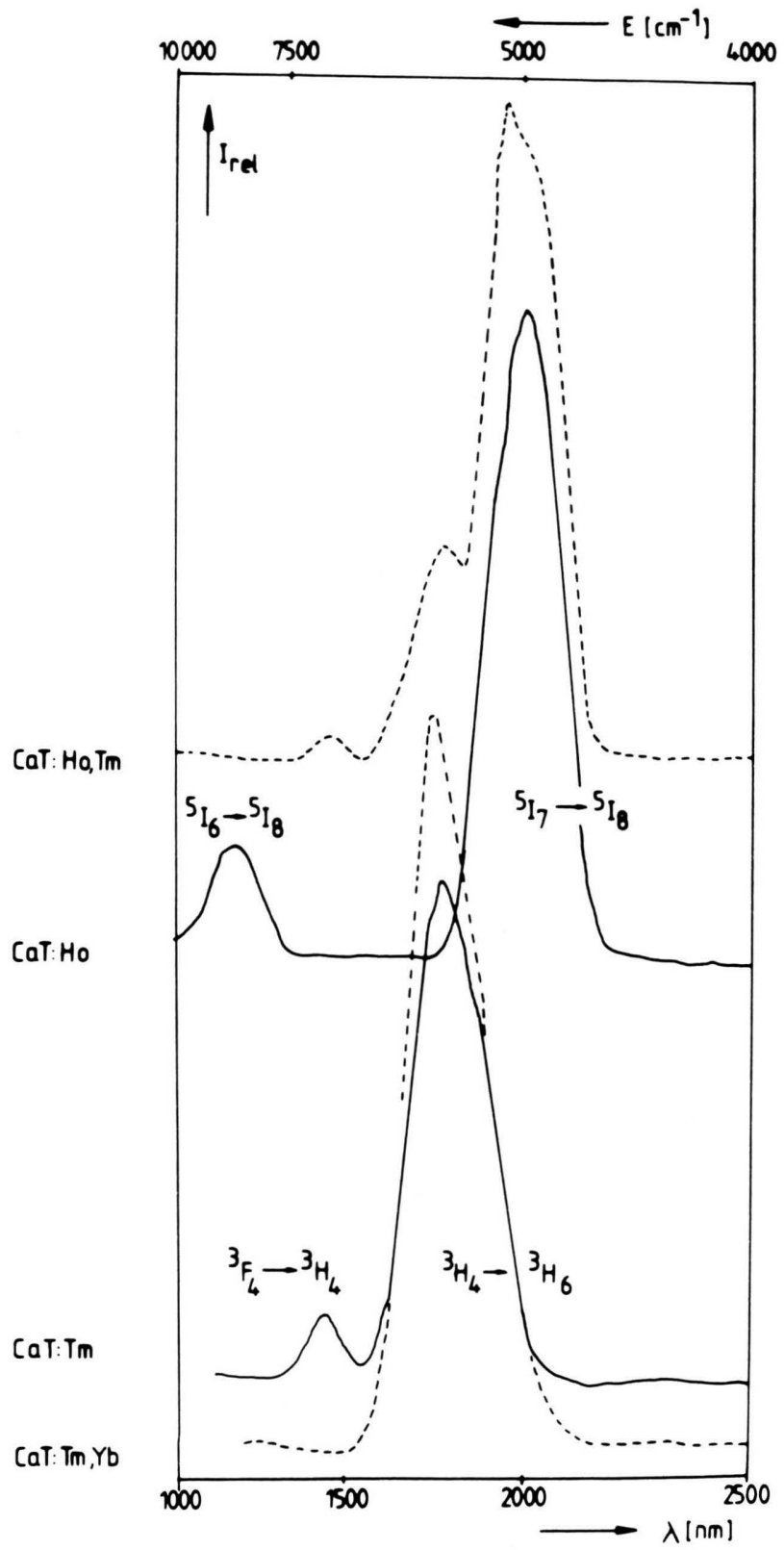

Abb. 5. Emissionsspektren von CaT:Ho, Tm $(x=0,05$; $\left.y=0,15 ; v_{\mathrm{A}} \sim 13500 \mathrm{~cm}^{-1}\right)$ und CaT:Ho $\left(x=0,05 ; v_{\mathrm{A}} \sim\right.$ $22000 \mathrm{~cm}^{-1}$; gegenüber CaT:Ho, Tm vierfache Verstärkung) sowie von CaT: Tm $\left(x=0,05 ; v_{\mathrm{A}} \sim 13500 \mathrm{~cm}^{-1}\right)$ und $\mathrm{CaT}: \mathrm{Tm}, \mathrm{Yb} \quad\left(x=0,05 ; y=0,2 ; v_{\mathrm{A}} \sim 10500 \mathrm{~cm}^{-1}\right.$; gleiche Verstärkung wie für CaT:Tm).

tiver Sensibilisatoreinstrahlung ein jeweils entsprechendes Emissionsverhalten wie für $\mathrm{CaT}: \mathrm{Ln}, \mathrm{Ln}^{\prime}$.

Ein Vergleich der bei Sensibilisator-Anregung erhaltenen Emissionsspektren von $\mathrm{CaT}: \mathrm{Ln}, \mathrm{Ln}^{\prime}$ mit den bei Aktivator-Einstrahlung beobachteten Spek- 
tren CaT:Ln zeigt für die Kombinationen $\mathrm{Ho} / \mathrm{Tm}$ und $\mathrm{Tm} / \mathrm{Yb}$ charakteristische Unterschiede (Abbildung 5). Gegenüber CaT:Ho treten für $\mathrm{CaT}: \mathrm{Ho}, \mathrm{Tm}$ zwar die beiden Tm-Übergänge ${ }^{3} \mathrm{H}_{4} \rightarrow{ }^{3} \mathrm{H}_{6}$ und ${ }^{3} \mathrm{~F}_{4} \rightarrow{ }^{3} \mathrm{H}_{4}$ (s.o.) hinzu, dagegen fehlt die höherfrequente $\mathrm{Ho}^{3+}$-Emission ${ }^{5} \mathrm{I}_{6} \rightarrow{ }^{5} \mathrm{I}_{8}$ bei $8500 \mathrm{~cm}^{-1}$. Im Emissionsspektrum von $\mathrm{CaT}: \mathrm{Tm}, \mathrm{Yb}$ läßt sich der $\mathrm{Tm}^{3+}$-Übergang ${ }^{3} \mathrm{~F}_{4} \rightarrow{ }^{3} \mathrm{H}_{4}\left(\sim 6800 \mathrm{~cm}^{-1}\right)$ nicht beobachten, welcher für $\mathrm{CaT}: \mathrm{Tm}$ vorhanden ist.

Bei Temperaturerniedrigung auf $77 \mathrm{~K}$ sinkt $I_{\text {rel }}$ für CaT: $L n, L n^{\prime}$ im IR ab, während im sichtbaren Gebiet ein Intensitätszuwachs auftritt.

\section{Diskussion}

Bei Aktivierung von CaT mit dreiwertigen Seltenen Erden ist mit Ausnahme von $\mathrm{Ln}^{3+}=\mathrm{Eu}$ im Gebiet von $\sim 40000 \mathrm{~cm}^{-1}$ eine Anregung allein über $4 \mathrm{f} \rightarrow 4 \mathrm{f}$-Übergänge möglich. Eine Beteiligung von Wirtsgitterbestandteilen an der Energieaufnahme wird nicht beobachtet. Für CaT:Eu läßt sich zusätzlich eine Anregung über den CT vom Sauerstoff zum $\mathrm{Eu}^{3+}$ erzielen. Mit $\sim 33000 \mathrm{~cm}^{-1}$ liegt dieser gegenüber der isotypen $\mathrm{W}^{6+}$-Verbindung $\mathrm{Ca}_{3} \mathrm{La}_{2} \mathrm{~W}_{2} \mathrm{O}_{12}$ : $\mathrm{Eu}\left(\sim 29000 \mathrm{~cm}^{-1}\right.$ [9] $)$ bei höheren Energien. Aufgrund der, auf das weitgehende Fehlen von $\pi$-Bindungen zurückzuführenden, geringen Stärke der $\mathrm{Te}^{6+}-\mathrm{O}$ - gegenüber $\mathrm{W}^{6+}-\mathrm{O}$-Bindungen und der damit einhergehenden Verstärkung des auf das $\mathrm{Eu}^{3+}$ wirkenden Felds der Sauerstoffliganden, ist dieser Effekt zu erwarten.

In den Verbindungen CaT: Ln liegt die Aktivatorkonzentration bei etwa $0,2 \cdot 10^{21} \mathrm{Ln}^{3+}$-Ionen pro $\mathrm{cm}^{3}$ $(x=0,05)$ bzw. bei $0,4 \cdot 10^{21} \mathrm{Ln}^{3+}{ }_{-}$Ionen pro $\mathrm{cm}^{-3}$ $(x=0,1)$, der durchschnittliche Ln-Ln-Abstand liegt bei $\sim 17 \AA(x=0,05)$ bzw. $14 \AA(x=0,1)$. Bei Einbau des gleichen Anteils an Sensibilisator $\left(\mathbf{L n}^{\prime}\right)$ beträgt der mittlere Ln-Ln'-Abstand etwa $14 \AA$ $(x=y=0,05)$ bzw. $11 \AA(x=y=0,1)$, so daß die Wahrscheinlichkeit einer Austauschkopplung bzw. von multipolaren Wechselwirkungen stark reduziert ist. Wird als Sensibilisator $\mathrm{Yb}^{3+}$ verwendet, ergibt sich im Bereich der $4 \mathrm{f}$-Terme jeweils nur ein Transfer-Weg [7], der das energetisch relativ niedrig liegende ${ }^{2} \mathrm{~F}_{5 / 2}$ Multiplett miteinbezieht:

$$
\begin{aligned}
& \mathrm{Yb}^{3+} \rightarrow \mathrm{Ho}^{3+}: \\
& { }^{2} \mathrm{~F}_{5 / 2}\left(\sim 9900 \mathrm{~cm}^{-1} \rightarrow{ }^{5} \mathrm{I}_{6} \quad\left(\sim 8500 \mathrm{~cm}^{-1}\right),\right.
\end{aligned}
$$

$$
\begin{aligned}
& \mathrm{Yb}^{3+} \rightarrow \mathrm{Tm}^{3+}: \\
& { }^{2} \mathrm{~F}_{5 / 2}\left(\sim 9900 \mathrm{~cm}^{-1}\right) \rightarrow{ }^{3} \mathrm{H}_{5}\left(\sim 8300 \mathrm{~cm}^{-1}\right), \\
& \mathrm{Nd}^{3+} \rightarrow \mathrm{Yb}^{3+}: \\
& { }^{4} \mathrm{~F}_{3 / 2}\left(\sim 10900 \mathrm{~cm}^{-1}\right) \rightarrow{ }^{2} \mathrm{~F}_{5 / 2}\left(\sim 9900 \mathrm{~cm}^{-1}\right) .
\end{aligned}
$$

In allen Fällen besteht zwischen Sensibilisatorund Aktivatorterm keine Resonanz. Das Aktivatorniveau ist um $1000-1600 \mathrm{~cm}^{-1}$ abgesenkt. Beim Transferproze $\beta$ muß die Energiedifferenz in Form von Phononen an das Gitter abgegeben werden. Diese können von den oktaedrischen $\mathrm{TeO}_{6}$-Gruppen des Wirtsgitters aufgenommen werden. Die Valenzschwingungen liegen bei $\sim 720 \mathrm{~cm}^{-1}$ ( $v_{1}$-Gebiet), $\sim 570 \mathrm{~cm}^{-1}\left(v_{2}\right)$ und $\sim 670 \mathrm{~cm}^{-1}\left(v_{3}\right)$; Deformation $\sim 430 \mathrm{~cm}^{-1}\left(v_{4}\right)$ und $450 \mathrm{~cm}^{-1}\left(v_{5}\right)[10]$.

Für die Kombination $\mathrm{Tm} / \mathrm{Yb}$ zeigt das bei $\mathrm{Yb}^{3+}$ Einstrahlung erhaltene Emissionsspektrum, daß die Energie über den ${ }^{2} \mathrm{~F}_{5 / 2}$-Term von $\mathrm{Yb}^{3+}$ zum $\mathrm{Tm}^{3+}$ geführt wird, denn es tritt ausschließlich die energiearme $\mathrm{Tm}^{3+}$-Emission ${ }^{3} \mathrm{H}_{4} \rightarrow{ }^{3} \mathrm{H}_{6}$ auf (Abbildung 5). Bei Abwesenheit des Sensibilisators (CaT: Tm) fließt die Energie im $\mathrm{Tm}^{3+}$-Termsystem und es erfolgt neben ${ }^{3} \mathrm{H}_{4} \rightarrow{ }^{3} \mathrm{H}_{6}$ auch eine Emission von einem energetisch höher liegenden $\mathrm{Tm}^{3+}$-Term ${ }^{3} \mathrm{~F}_{4}\left(\sim 12500 \mathrm{~cm}^{-1}\right)$, welcher für CaT: Tm, Yb bei $\mathrm{Yb}^{3+}$-Einstrahlung nicht mehr erreichbar ist.

Für die Kombination Ho/Er ist mit einem Energietransfer ${ }^{4} \mathrm{I}_{13 / 2} \quad\left(\mathrm{Er} ; \sim 6500 \mathrm{~cm}^{-1}\right) \rightarrow{ }^{5} \mathrm{I}_{7} \quad(\mathrm{Ho}$; $\sim 5000 \mathrm{~cm}^{-1}$ ) zu rechnen [7]. Allerdings bestehen aufgrund der dichten, sowohl für $\mathrm{Ho}^{3+}$ als auch $\mathrm{Er}^{3+}$ beobachteten Termfolge zahlreiche weitere wechselseitige Transfer-Wege. Für CaT:Ho, Er bleibt die Energieübertragung unvollständig. Auf eine Beteiligung höher angeregter Terme am Energietransfer könnte die Beobachtung hindeuten, daß bei Er-Anregung $\left({ }^{4} \mathrm{I}_{15 / 2} \rightarrow{ }^{4} \mathrm{G}_{11 / 2}\right)$ neben der HoEmission ${ }^{5} \mathrm{I}_{7} \rightarrow{ }^{5} \mathrm{I}_{8}$ auch der energiereichere Übergang ${ }^{5} \mathrm{I}_{6} \rightarrow{ }^{5} \mathrm{I}_{8}$ auftritt.

Bei der Kombination Ho/Tm kann die Energieübertragung von ${ }^{3} \mathrm{H}_{4}\left(\mathrm{Tm} ; \sim 5500 \mathrm{~cm}^{-1}\right)$ auf ${ }^{5} \mathrm{I}_{7}$ (Ho; $\sim 5000 \mathrm{~cm}^{-1}$ ) erfolgen [7]. Dieser TransferWeg ergibt sich aus dem bei $\mathrm{Tm}^{3+}$-Einstrahlung erhaltenen Emissionsspektrum von CaT:Ho, Tm (Abb. 5): von den Ho-Übergängen wird allein ${ }^{5} \mathrm{I}_{7} \rightarrow{ }^{5} \mathrm{I}_{8}$ beobachtet, während die energiereichere Bande ${ }^{5} \mathrm{I}_{6} \rightarrow{ }^{5} \mathrm{I}_{8} \quad\left(\sim 8500 \mathrm{~cm}^{-1}\right)$ im Gegensatz zu CaT:Ho fehlt, da der Term ${ }^{5} \mathrm{I}_{6}$ bei dem Transfer ${ }^{3} \mathrm{H}_{4} \rightarrow{ }^{5} \mathrm{I}_{7}$ nicht mehr zu erreichen ist. Insgesamt bleibt in CaT: Ho, Tm der Energietransfer $\mathrm{Tm} \rightarrow \mathrm{Ho}$ jedoch unvollständig, da auch Tm-Terme zur Emission gelangen. 
Ein Vergleich der bei selektiver Anregung erzielten relativen Emissionsintensitäten zwischen den sensibilisierten (CaT:Ln,Ln') und nicht sensibilisierten Verbindungen (CaT:Ln; jeweils für den gleichen Aktivator Ln bei konstanter Konzentration $(x=0,05))$ zeigt, daß für alle $\mathrm{Ln} / \mathrm{Ln}^{\prime}-$ Kombinationen die Einstrahlung in den stärksten Ln-Übergang zu einer praktisch gleich intensiven IR-Emission führt wie bei CaT:Ln. Der in CaT: $\mathrm{Ln}$ vorhandene Anregungsweg für die Ln-Emission wird durch den Einbau der Sensibilisatoren $\mathbf{L n}^{\prime}$ nicht betroffen. Bei den verwendeten Sensibilisatorkonzentrationen hat die Einstrahlung in den stärksten Ln'-Übergang eine gesteigerte Emissionsintensität zur Folge (CaT:Ho, Er Faktor 1,5; CaT:Ho, Tm Faktor 4; CaT:Ho, $\mathrm{Yb} \sim$ Faktor 3,5) bzw. erbringt eine leichte Verrin-

[1] H.-D. Autenrieth u. S. Kemmler-Sack, Mater. Chem. Phys. 12, 437 (1985).

[2] B. Köngeter u. S. Kemmler-Sack, Z. Naturforsch. 39 a, 490 (1984).

[3] B. Köngeter u. S. Kemmler-Sack, Mater. Chem. Phys. 11, 495 (1984).

[4] J. M. Longo, L. Katz u. R. Ward, Inorg. Chem. 3, 205 (1964).

[5] R. Otto u. S. Kemmler-Sack, Ber. Bunsenges. Phys. Chem. 88, 931 (1984).

[6] W. H. Fonger u. C. W. Struck, J. Chem. Phys. 52, 6344 (1970). gerung $(\mathrm{CaT}: \mathrm{Tm}, \mathrm{Yb} \sim$ Faktor 0,8). Bei breitbandiger Anregung führen die durch den Ln'-Einbau für $C a T: L n, L^{\prime}$ eröffneten neuen Anregungswege gegenüber CaT: Ln stets zu einer Steigerung der LnEmission. Wie für $\mathrm{CaT}: \mathrm{Ho}, \mathrm{Er}, \mathrm{Yb}$ und insbesondere $\mathrm{CaT}: \mathrm{Ho}, \mathrm{Er}, \mathrm{Tm}, \mathrm{Yb}$ gezeigt werden konnte, ist dieser Effekt beim gleichzeitigen Einbau mehrerer Sensibilisatoren mit über einen breiten Spektralbereich gestreuten Anregungsmaxima am größten.

Wir danken dem Bundesministerium für Forschung und Technologie für die Unterstützung der Untersuchung. Frau A. Ehmann und Frau R. Hüpper danken wir für ihre Hilfe. Die Arbeit wurde durch die Deutsche Forschungsgemeinschaft und den Verband der Chemischen Industrie gefördert.

[7] A. A. Kaminskii, Laser Crystals, Springer Series in Optical Sciences, Springer Verlag, Berlin 1981.

[8] M. J. Weber in: Lanthanide and Actinide Chemistry and Spectroscopy, p. 275 (ed. N. M. Edelstein) ACS Symposium Series 113, American Chemical Society, Washington D.C. 1980.

[9] H.-D. Autenrieth und S. Kemmler-Sack, Phys. Stat. Sol. (a) 83,631 (1984).

[10] H.-D. Autenrieth, unveröffentlichte Ergebnisse. 